\title{
Erratum to: Dissipative quantum backflow
}

\author{
S. V. Mousavi ${ }^{1, a}$, S. Miret-Artés ${ }^{2, b}$ \\ ${ }^{1}$ Department of Physics, University of Qom, Ghadir Blvd., Qom 371614-6611, Iran \\ 2 Instituto de Física Fundamental, Consejo Superior de Investigaciones Científicas, Serrano 123, \\ 28006 Madrid, Spain
}

Published online: 14 August 2020

(C) Società Italiana di Fisica and Springer-Verlag GmbH Germany, part of Springer Nature 2020

\section{Erratum to: Eur. Phys. J. Plus (2020) 135:324 https://doi.org/10.1140/epjp/s13360-020-00336-5}

In a recent article [1] where quantum backflow for open systems within the Caldirola-Kanai (CK) and Caldeira-Leggett (CL) frameworks is considered, we have realized that, although the reported results in the CL context are correct for one and two Gaussian wave packets, our interpretation as quantum backflow was somehow hasty. As mentioned in this article, quantum backflow which takes place in the free evolution regime is seen when a purely positive momentum distribution leads to negative fluxes during some time intervals and at some positions. We only computed initial momentum distributions, but our systems are interacting with the environment through a frictional force and thermal noise. Thus, to really confirm that the effects found at later times are due to quantum backflow, we should also have provided the momentum distribution as a function of time.

Within the context of the CL master equation, when a quantum system is initially described by the pure state given by Eq. (15) in the momentum space, the probability for obtaining a negative value in a measurement of momentum at time $t$, when no external interaction potential is present, is given by

$$
\begin{aligned}
\operatorname{Prob}(p<0, t)= & \frac{1}{2} N^{2}\left\{\operatorname{erfc}\left[\frac{p_{0 a} e^{-2 \gamma t}}{\sqrt{2 f(t)}}\right]+\alpha^{2} \operatorname{erfc}\left[\frac{p_{0 b} e^{-2 \gamma t}}{\sqrt{2 f(t)}}\right]\right. \\
& \left.+2 \alpha e^{-\left(p_{0 a}-p_{0 b}\right)^{2} / 8 \sigma_{p}^{2}} \cos \theta \operatorname{erfc}\left[\frac{\left(p_{0 a}+p_{0 b}\right) e^{-2 \gamma t}}{2 \sqrt{2 f(t)}}\right]\right\} \\
f(t)= & \left(\sigma_{p}^{2}-\frac{D}{2 \gamma}\right) e^{-4 \gamma t}+\frac{D}{2 \gamma}
\end{aligned}
$$

The arguments of all complementary error functions in this equation are decreasing functions of time. Thus, $\operatorname{Prob}(p<0, t)$ increases with time and takes the asymptotic value 0.5 . Therefore, the probability of finding the system in the region $x<0$ also grows with time. In this sense, we cannot speak about quantum backflow nor for a single Gaussian wave packet,

The original article can be found online at https://doi.org/10.1140/epjp/s13360-020-00336-5.

\footnotetext{
a e-mail: vmousavi@qom.ac.ir (corresponding author)

b e-mail: s.miret@iff.csic.es
} 
$\alpha=0$, nor for the superposed state, $\alpha \neq 0$. The backflow exists, but it is due to the presence of the environment, even if the external potential is zero.

On the contrary, in the free evolution regime and in the CK approach, $\operatorname{Prob}(p<0, t)$ is independent of time regardless of the shape of the initial wave function. Thus, for example, if the initial state formed by a linear combination of two Gaussian wave packets has negligible negative momentum contribution, its time evolution too. The dissipative quantum backflow effect is clearly seen in this framework.

\section{References}

1. S.V. Mousavi, S. Miret-Artés, Eur. Phys. J. Plus 135, 324 (2020) 